Abstract ID: 134

\title{
Anti-fertility potential of Trigonella foenum-graecum in comparison to oral contraceptive pills
}

\author{
Ahmed Kaid Naji Allow | Ayah Rebhi Hilles | Zainab Yousef | Norbaiyah Mohamed Bakrim | Belqees \\ Ahmed | Anil Kumar Saxena \\ Department of Basic Medical Sciences, Kulliyyah of Medicine, International Islamic University Malaysia
}

Introduction: This study examines the anti-fertility effects of Trigonella foenumgraecum (fenugreek) seeds extract in the reproductive system of female rats in comparison to combined oral contraceptive pills (COCPs). Methods: Thirty two female Sprague Dawley rats of 8 weeks old were divided into four groups $(A, B, C$ and $D)$. All female rats were allowed to mate with male rats. Group A is a positive control and not given any treatment. Group B is a negative control group. Group $C$ and $D$ were treated with $0.05 \mathrm{mg} / \mathrm{kg}$ body weight of COCPs and $750 \mathrm{mg} / \mathrm{kg}$ body weight of fenugreek seed aqueous (FSA) extract for 15 days respectively. Blood samples were subsequently taken on the proestrous phase to evaluate the serum levels of follicular stimulating hormone (FSH). The embryo implantation rate was studied in all groups. Results: Administration of $750 \mathrm{mg} / \mathrm{kg}$ FSA extract and COCPs both resulted in decreased FSH serum levels, to $25.62 \mathrm{ng} / \mathrm{ml}$ and $71.56 \mathrm{ng} / \mathrm{ml}$ respectively $(p<0.01)$. The embryo implantation rate was zero in both FSA extract and COCPs treated groups. Conclusions: Anti-fertility effects of FSA extract and COCPs are potentially similar in terms of their ability to reduce FSH serum levels and inhibit implantation.

KEYWORDS: Anti-fertility, Trigonella foenum-graecum, COCPs, Endometrial Thickness 